\title{
Statistical aspects of a model for interpersonal attraction
}

\author{
PETER H. SCHÖNEMANN, DONN BYRNE, and PAUL A. BELL \\ Purdue University, West Lafayette, Indiana 47907
}

\begin{abstract}
An interpersonal attraction model which Byrne (1971) and his co-workers (Byrne \& Nelson, 1965; Byrne \& Rhamey, 1965) developed in conjunction with a series of interrelated empirical experiments is reinterpreted as a multiple regression model.
\end{abstract}

For more than a decade Byrne and his co-workers (Byrne, 1971; Byrne, London, \& Griffitt, 1968; Byrne \& Nelson, 1965; Byrne \& Rhamey, 1965) have been employing a general paradigm in empirical research which states that interpersonal attraction, if suitably quantified in a measure $c_{i j}$, is a positive linear function of the proportion of attitudes $\mathrm{p}_{\mathrm{ij}}$ shared by two people $S_{i}, S_{j}$ :

$$
c_{i j}=a p_{i j}+b .
$$

Approximately the same linear relationship

$$
\mathrm{c}_{\mathrm{ij}}=5.4 \mathrm{p}_{\mathrm{ij}}+6.6
$$

held up across a variety of experiments which differed in design and subject population. The similarity argument $\mathrm{p}_{\mathrm{ij}}$ had been defined originally (Byrne \& Nelson, 1965) as the normalized counting measure on the set of attitudes shared by both subjects, that is,

$$
p_{i j}^{*}=m^{+} /\left(m^{+}+m^{-}\right),
$$

where $\mathrm{m}^{+}$denotes the number of attitude items to which $S_{i}$ and $S_{j}$ respond the same way and $m^{-}$is the number of remaining items on which they disagree. Thus, $\mathrm{p}_{\mathrm{ij}}^{*}$ is simply the proportion of attitudes shared by $S_{i}$ and $S_{j}$. When it later became apparent that agreement on some items affected the attraction response more strongly than agreement on others, Byrne and Rhamey (1965) generalized the unweighted $p_{i j}^{*}$ to a weighted proportion:

$$
\mathrm{p}_{\mathrm{ij}}=\mathrm{w}^{+} /\left(\mathrm{w}^{+}+\mathrm{w}^{-}\right) .
$$

Each of the $m$ items is now characterized by a weight $\mathrm{w}_{\mathrm{k}} ; \mathrm{w}^{+}$is the sum of the weights agreed on by both $S_{i}$ and $S_{j}$, and $w^{-}$is the sum of the weights for the

This research was supported in part by National Science Foundation Grant GS-40329 to Donn Byrne and in part by Grant MH 28687 from NIMH to Peter Schőnemann. Requests for reprints should be sent to Peter H. Schōnemann, Department of Psychological Sciences, Purdue University, West Lafayette, Indiana 47907. remaining items on which they disagree. Since the $\mathrm{p}_{\mathrm{ij}}$ in Formula 1.4 contains the $\mathrm{p}_{\mathrm{ij}}^{*}$ in Formula 1.3 as a special case when all the $\mathrm{m} \mathrm{w}_{k}$ are equal, we will focus on this slightly more general formulation of the attraction paradigm:

$$
c_{i j}=a^{+} /\left(w^{+}+w^{-}\right)+b .
$$

In previous research the weights $w_{k}$ were typically obtained in a separate scaling experiment where a number of judges were asked to scale the importance of the $\mathrm{m}$ attitude items (Byrne et al., 1968; Clore \& Baldridge, 1968). As we will show, such separate scaling experiments are, strictly speaking, unnecessary because the weights $w_{k}$ can be estimated from the $c_{i j}$ and the attitude responses of the subjects. The $m$ weights and the parameters $a, b$ can be interpreted as regression weights. The regression weights can be estimated by the familiar multiple regression techniques which, furthermore, provide optimal statistical tests for hypotheses about these weights within the framework of the general linear model (GLM). We will further show that the Byrne-Rhamey formula (1.5) can be interpreted as a distance model which bears some resemblance to a currently popular individual difference model for multidimensional scaling, the subjective metrics model proposed by Bloxom (1968), Carroll and Chang (1970), and Horan (1969). The simple attraction paradigm (Formula 1.5) thus has richer structure than might appear on superficial examination.

\section{THE BYRNE-RHAMEY FORMULA AS A DIST ANCE MODEL}

In a typical attraction experiment, $m$ binary attitude items $A_{1} \ldots A_{k} \ldots A_{m}$ are presented to $p$ subjects, instructing them to indicate which items they endorse. The experimenter may construct $\mathrm{q}$ more fictitious subjects ("bogus strangers") by prescribing which attitude items such a stranger has endorsed. One thus obtains $a(p+q)$ by $m$ matrix of binary responses. If we encode these data numerically in a matrix $A=\left(a_{i k}\right)$ with $a_{i k}=1$ if subject $S_{i}$ endorses the kth attitude item, and $a_{i k}=-1$ if he opposes it, the ith row in A might be 


$$
a_{i}{ }^{\prime}=(1,1,-1,1,-1)
$$

and the jth row

$$
a_{j}{ }^{\prime}=(1,-1,-1,-1,1) .
$$

Here, the ith subject, $S_{i}$, has endorsed the first, second, and fourth attitude items and the jth bogus stranger has been made to endorse the first and fifth. There are altogether $m=5$ items and the subject agrees with the stranger on the first and third, that is, on $\mathrm{m}^{+}=2$ items. The unweighted proportion $\mathrm{p}_{\mathrm{ij}}^{*}$ in Formula 1.3 is thus .4. If we assign "importance weights" $\mathrm{w}_{\mathrm{k}}=2$, $1,4,1,2$ to the five items, the weighted proportion $\mathrm{p}_{\mathrm{ij}}$ in Formula 1.4 would be $\mathrm{p}_{\mathrm{ij}}=.6$.

We then obtain an "attraction score" $c_{i j}$ by asking subject $S_{i}$ to indicate on some rating scale (e.g., the Interpersonal Judgment Scale, or IJS, which varies from 2 to 14) the extent to which he feels "attracted" to stranger $S_{j}$, given $a_{j}^{\prime}$, that is, his score profile on the attitude items.

In this section we show that the Byrne-Rhamey (Formula 1.5) model has an interesting geometrical interpretation when all the weights $\mathbf{w}_{\mathbf{k}}$ are positive. The weighted proportion $\mathrm{p}_{\mathrm{ij}}$ in Formula 1.4 can be written

$$
\mathrm{p}_{\mathrm{ij}}=\mathrm{w}^{+} /\left(\mathrm{w}^{+}+\mathrm{w}^{-}\right)=\left(\alpha_{\mathrm{i}}^{\prime} \mathrm{D} \alpha_{\mathrm{j}}+\mathrm{J}^{\prime} \mathrm{DJ}\right) / 2 \mathrm{~J}^{\prime} \mathrm{DJ}
$$

where $D=\operatorname{diag}\left(w_{1}, \ldots, w_{k}, \ldots, w_{m}\right)$ is the diagonal matrix of weights and $J^{\prime}=(1,1, \ldots, 1)$ is a vector of $\mathrm{m}$ ones, so that

$$
\mathbf{J}^{\prime} \mathrm{DJ}=\sum_{\mathbf{k}}^{\mathrm{m}} \mathbf{w}_{\mathbf{k}} \cdot
$$

Hence, the response $c_{i j}$ is a linear function of the scalar product $\alpha_{i}^{\prime} D \alpha_{j}$ and the Byrne-Rhamey formula (2.1) can be interpreted as a scalar product model.

But since all row vectors $\alpha_{i}^{\prime}$ in $A$ are of equal (euclidean) length $\left|\alpha_{\mathrm{i}}\right|=\mathrm{m}^{1 / 2}$, it can also be interpreted as a distance model. The weighted proportions $\mathrm{p}_{\mathrm{ij}}$ are linear functions of squared (elliptical) distances:

$$
\begin{aligned}
\mathrm{p}_{\mathrm{ij}} & =\left(\alpha_{\mathrm{i}}^{\prime} \mathrm{D} \alpha_{\mathrm{j}}+\mathrm{J}^{\prime} \mathrm{DJ}\right) / 2 \mathrm{~J}^{\prime} \mathrm{DJ} \\
& =1-\left[\left(\alpha_{\mathrm{i}}-\alpha_{\mathrm{j}}\right)^{\prime} \mathrm{D}\left(\alpha_{\mathrm{i}}-\alpha_{\mathrm{j}}\right)\right] / 4 \mathrm{~J}^{\prime} \mathrm{DJ} \\
& =1-\mathrm{d}^{2}{ }_{\mathrm{ij}} / \mathrm{d}^{2} \max
\end{aligned}
$$

where

$$
\mathrm{d}^{2}{ }_{\mathrm{ij}}=\left(\alpha_{\mathrm{i}}-\alpha_{\mathrm{j}}\right)^{\prime} \mathrm{D}\left(\alpha_{\mathrm{i}}-\alpha_{\mathrm{j}}\right)
$$

is a (squared) elliptical distance and

$$
\mathrm{d}^{2}{ }_{\max }=\max _{\alpha_{i}, \alpha_{j}}\left[\left(\alpha_{i}-\alpha_{j}\right)^{\prime} \mathrm{D}\left(\alpha_{\mathrm{i}}-\alpha_{\mathrm{j}}\right)\right]=4 \mathrm{~J}^{\prime} \mathrm{DJ}
$$

is its maximum over the set of admissible $\alpha_{i}, \alpha_{j}$ as defined above. The Byrne-Rhamey formulation thus becomes

$$
c_{i j}=a\left(1-d_{i j}^{2} / d_{\text {max }}^{2}\right)+b .
$$

This means it can be interpreted as a distance model, as long as all $w_{k}$ are positive. If at least two $w_{k}$ are distinct, it employs the weighted "elliptical" distance function, Formula 2.4 .

Such elliptical distances also occur in the subjective metrics model proposed by Bloxom (1968), Carroll and Chang (1970), and Horan (1969) which was solved algebraically by Schönemann (1972). This model states that the distances $d_{i j . r}$ for all pairs of $p$ stimuli $(i, j=1, p)$ in $N$ subject-specific matrices $\Delta_{\mathrm{r}}=\left(\mathrm{d}_{\mathrm{ij} . \mathrm{r}}\right)(\mathrm{r}=1, \mathrm{~N})$ can be resolved as

$$
\begin{gathered}
\left.\Delta_{\mathrm{r}}(2)=\mathrm{d}_{\mathrm{ij} . \mathrm{r}}^{2}\right)=\left[\left(\alpha_{\mathrm{i}}-\alpha_{\mathrm{j}}\right)^{\prime} \mathrm{D}_{\mathrm{r}}\left(\alpha_{\mathrm{i}}-\alpha_{\mathrm{j}}\right)\right] \\
\mathrm{i}, \mathrm{j}=1, \mathrm{p} ; \mathrm{r}=1, \mathrm{~N}
\end{gathered}
$$

where the "subjective metric" matrices $D_{r}$ are diagonal and contain $\mathrm{m}$ subjects' specific weights $\mathrm{w}_{1 \mathrm{r}}, \ldots$, $\mathrm{w}_{\mathrm{kr}}, \ldots, \mathrm{w}_{\mathrm{mr}}$. This model is superficially similar to the Byrne-Rhamey model, especially once the latter is generalized into an individual difference model. This is easily accomplished by introducing subject-specific weights $\mathrm{w}_{\mathrm{rk}}, \mathrm{r}=1, \mathrm{~N}$ (see Schönemann et al., 1975 for details).

There are, however, important differences. The Byrne-Rhamey formulation is tied to a clear cut data base. We know exactly how to conduct an experiment to obtain the protocol information, and there are plausible psychological reasons why it might describe such data. In contrast, the proponents of the subjective metrics model leave the data base vague and largely unspecified. Prior transformations of the data may further weaken the relation between model and data. More importantly, the coordinate vectors $\alpha_{\mathrm{i}}$ are empirically given in the Byrne-Rhamey model. Only the parameters $a, b$ and the $m$ weights $w_{k}$ need be estimated, not both $D$ and $A=\left(\alpha_{i}\right)$, as in the subjective metrics model. This means that the Byrne-Rhamey formula (2.1) is a stronger model which, on the one hand, is more easily falsified and, on the other, is more informative and predictive when it fits.

One advantage of this distance interpretation of the attraction model is the associated geometry, which is depicted in Figure 1. There we deal with two attitude items, $A_{1}$ and $A_{2}$, which define a basis for the $m$ space of the response vectors $\alpha_{1}$. If $m=2$, as in the diagram, this space is a plane, and there are altogether $2^{\mathrm{m}}=4$ distinct possible response vectors $\alpha_{1}$. They are all of equal length $\left|\alpha_{i}\right|=\sqrt{\mathrm{m}}$ and thus lie on the surface of an $\mathrm{m}$ sphere with radius $\sqrt{\mathrm{m}}$ or, equivalently, are the $2^{\mathrm{m}}$ corners of an $\mathrm{m}$ cube inscribed in this sphere. In the plane this is a square with sides of Length 2 inscribed in a circle of radius $\sqrt{2}$. As long as all weights are equal, the model states that the dependent variable $\mathrm{c}_{\mathrm{ij}}$ is a linear function of the (squared euclidean) distance between the endpoints of the two response vectors $\alpha_{i}$ and $\alpha_{j}$, for example, the squared length of the dotted 


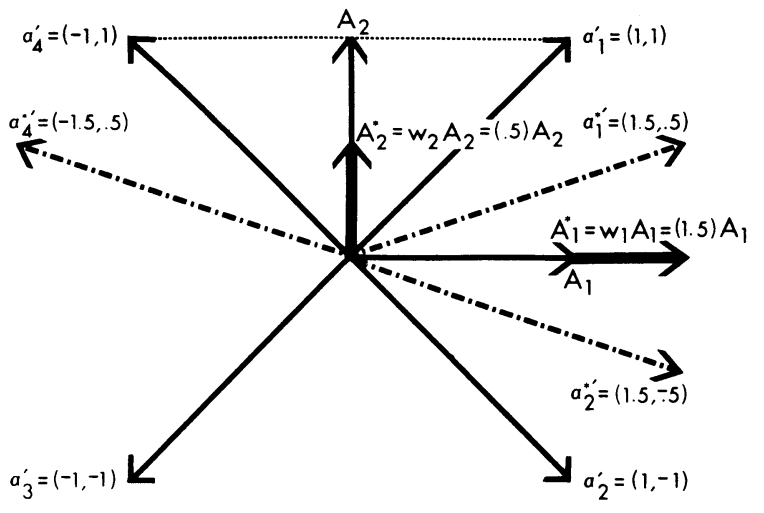

Figure 1. Geometry of the Byrne-Rhamey model.

line segment between $\alpha_{1}$ and $\alpha_{4}$ in the diagram. For $\mathrm{m}=2$, the possible distances in the unweighted case are thus 0 (e.g., $\alpha_{1}$ and $\alpha_{1}$ ), 2 (e.g., $\alpha_{1}$ and $\alpha_{4}$ ), and $2 \sqrt{2}$ (e.g., $\alpha_{1}$ and $\alpha_{3}$ ).

In the more general weighted case, the coordinate vector $A_{k}$ is shrunk or stretched in length according to whether the corresponding $w_{k}$ is smaller or larger than unity. Therefore, all projections on the $A_{k}$ of the response vectors $\alpha_{i}$ shrink or stretch in length. The responses are now represented by the oblique vectors $\alpha_{i}^{*}$ which lie on an $m$ sphere with radius

$$
\sqrt{\sum_{\mathrm{w}}} \mathrm{k}^{2}
$$

We could view them as the $2^{\mathrm{m}}$ corners of a parallelepiped inscribed in an ellipsoid. Two response vectors are inclined toward each other when they enclose a more heavily weighted item, for example, $\alpha_{1}^{*}$ and $\alpha_{2}^{*}$ in the diagram. Therefore the distance between their endpoints will be less than if they enclose a less important item (e.g., $\alpha_{1}^{*}$ and $\alpha_{4}^{*}$ ) and the attraction response $\mathrm{c}_{\mathrm{ij}}$ will be larger.

\section{THE BYRNE-RHAMEY MODEL AS A LINEAR MODEL}

Upon reviewing the weighted model $(2.1,2.2)$ in scalar product form,

$$
c_{i j}=a p_{i j}+b=\frac{a}{2 J^{\prime} D J} \sum_{k} a_{i k} a_{j k} w_{k}+\frac{a}{2}+b \quad(i \neq j)
$$

or in distance form, using Formula 2.3

$$
c_{i j}=a p_{i j}+b=a+b-\frac{a}{4 J^{\prime} D J} \sum_{k}\left(a_{i k}-a_{j k}\right)^{2} w_{k} \quad(i \neq j)
$$

it becomes clear that we are dealing in both cases with a multiple regression model, that is, a special case of the GLM. To see this for the scalar product formulation (3.1), we define

$$
\mu^{*}=\frac{a}{2}+b, w_{k}^{* *}=a w_{k} / 2 J ' D J, h_{i j k}^{*}=a_{i k} a_{j k}, H^{*}=\left(h_{j k}^{*}\right)
$$

Then Formula 3.1 becomes $c_{\mathrm{ij}}=\mu^{*}+\sum_{\mathrm{k}} \mathrm{h}_{\mathrm{j} \mathrm{jk}}^{*} \mathrm{w}_{\mathrm{k}}^{* *}$, or

$$
\gamma=\mathrm{H} \delta, \text { where } \delta^{\prime}=\left(\mu, \mathrm{w}_{\mathbf{1}}^{* *}, \ldots, \mathrm{w}_{\mathbf{m}}^{* *}\right), \mathrm{H}=\left(\mathrm{J}, \mathrm{H}^{*}\right) .
$$

To see it for the distance formulation (3.2) we define

$$
\mu=\mathrm{a}+\mathrm{b}, \mathrm{w}_{\mathrm{k}}^{*}=-\mathrm{aw}_{\mathrm{k}} / 4 \mathrm{~J}^{\prime} \mathrm{DJ}, \mathrm{g}_{\mathrm{j} k \mathrm{k}}^{*}=\left(\mathrm{a}_{\mathrm{ikk}}-\mathrm{a}_{\mathrm{jk}}\right)^{2}, \mathrm{G}^{*}=\left(\mathrm{g}_{\mathrm{jk}}^{*}\right) \text {. }
$$

Then Formula 3.2 reduces to $\mathrm{c}_{\mathrm{ij}}=\mu+\sum_{\mathrm{k}} \mathrm{g}_{\mathrm{jjk}}^{*} \mathrm{w}_{\mathrm{k}}^{*}$, or

$$
\gamma=\mathrm{G} \beta, \text { where } \beta^{\prime}=\left(\mu, \mathrm{w}_{1}^{*}, \ldots, \mathrm{w}_{\mathrm{m}}^{*}\right), \mathrm{G}=\left(\mathrm{J}, \mathrm{G}^{*}\right) .
$$

We can invoke in both cases the machinery of the GLM to obtain easily computed and statistically optimal estimates for the $\mathrm{m}+1$ parameters $\mu, \mathrm{w}_{\mathrm{i}}^{*}, \ldots, \mathrm{w}_{\mathrm{m}}^{*}$ of the weighted model in the fallible case, if we are willing to entertain the usual mild assumptions required by the GLM. Adopting Formula 3.6 as the expected value $\mathrm{E}(\gamma)$ of the response vector $\gamma=\left(c_{i j}\right)$, in the fallible case we add an error term $\epsilon$

$$
\gamma=\mathrm{G} \beta+\epsilon .
$$

The "design matrix" $\mathrm{G}$ is defined as in Formulas 3.5 and 3.6, and $\beta$ as in 3.6. The residual vector $\epsilon$ is assumed to contain $p+q$ random variables $e_{i}$ which jointly satisfy

$$
\mathrm{E}(\epsilon)=\phi, \operatorname{var}(\epsilon)=\sigma^{2} \mathrm{I}_{\mathrm{p}+\mathrm{q}} \text { for some } \sigma^{2}>0 .
$$

As is well known, this is all that is needed to claim that the least squares estimate (LSE) $\hat{\beta}$ be "minimum variance unbiased" among all linear estimates for $\beta$, as long as the design matrix $G$ is of full column rank (see e.g., Searle, 1966, Chap. 10, for a more thorough exposition of the GLM). This we shall have to assume, because if $\mathrm{G}$ were of deficient column rank we would lose "estimability" of $\beta$ [since $\beta$ in $\mathrm{E}(\gamma)=\mathrm{G} \beta$ would no longer be uniquely defined], and our primary objectives to estimate the parameters $a, b, w_{1}, \ldots, w_{m}$. Under the full rank condition we can compute the LSE $\hat{\beta}$ for $\beta$ by means of any standard multiple regression program (e.g., Wang \& Schönemann, 1970):

$$
\hat{\beta}=\left(G^{\prime} G\right)-1 G^{\prime} \gamma .
$$

It should be noted that, although the components $\mu, \mathrm{w}_{\mathrm{k}}^{*}$ in $\beta$, and their estimates are uniquely defined once $G$ is of full column rank, the actual weights $w_{k}$ of the attraction paradigm are defined only up to a multiplicative constant by Formula 3.5. This indeterminacy could be used to eliminate the slope parameter altogether by setting

$$
\mathrm{a}=4 \mathrm{~J}^{\prime} \mathrm{DJ}=4 \underset{\mathrm{k}}{\Sigma} \mathrm{w}_{\mathrm{k}},
$$

to identify the $w_{k}$. However, we prefer to retain the slope parameter a in order to obtain the unweighted attraction model $(1.1,1.3)$ from the weighted model (1.5) as a special case when the former cannot be rejected. In order to achieve comparability between the parameters of both models, we impose the identifiability constraint

$$
J^{\prime} \mathbf{D J}=\sum_{\mathrm{k}}^{\mathrm{m}} \mathrm{w}_{\mathrm{k}}=\mathrm{m} .
$$


This constraint eliminates the multiplicative indeterminacy. With this last step, our estimation task is completed. We now can solve Formula 3.5 for the parameters

$$
\mathrm{a}=-4 \sum_{\mathrm{k}}^{\mathrm{m}} \mathrm{w}_{\mathrm{k}}^{*}, \mathrm{~b}=\mu-\mathrm{a}, \mathrm{w}_{\mathrm{k}}=-4 \mathrm{mw}_{\mathrm{k}}^{*} / \mathrm{a}
$$

of the original attraction model in terms of the parameters of the regression model and their respective estimates.

In order to justify the usual $\mathrm{F}$ tests for testing hypotheses about $\beta$, we add a normality assumption

$$
\gamma \sim \mathrm{N}_{\mathrm{p}+\mathrm{q}}\left(\mathrm{G} \beta, \sigma^{2} \mathrm{I}\right) .
$$

With these assumptions we can test any "linear hypothesis," that is, hypotheses $\mathrm{H}_{\mathrm{o}}$ of the form

$$
\mathrm{H}_{\mathrm{o}}: \mathrm{Q}^{\prime} \beta=\theta
$$

for any given $k$ by $(m+1)$ "hypothesis matrix" $Q$ ' of full row rank $\mathrm{k}$ and any vector $\theta$ of constants. It is well known that in the full column rank case not only $\beta$ itself, but all linear functions $Q^{\prime} \beta$ are estimable and all linear hypotheses "testable" (see Searle, 1966; Seber, 1966; Wang \& Schönemann, 1970). The test statistic for such "linear hypotheses" is given by

$$
\mathrm{T}(\gamma)=\left(\mathrm{Q}^{\prime} \hat{\beta}-\theta\right)^{\prime}\left[\mathrm{Q}^{\prime}\left(\mathrm{G}^{\prime} \mathrm{G}\right)^{-1} \mathrm{Q}\right]^{-1}\left(\mathrm{Q}^{\prime} \hat{\beta}-\theta\right) / \hat{\sigma}^{2},
$$

where $\hat{\sigma}^{2}=(\gamma-\mathrm{G} \hat{\beta})^{\prime}(\gamma-\mathrm{G} \hat{\beta}) /(\mathrm{N}-\mathrm{r}), \mathrm{r}=\operatorname{rank}(\mathrm{G})=$ $\mathrm{m}+1, \mathrm{~N}=\mathrm{p}+\mathrm{q}$, and where $\mathrm{T}(\gamma)$ is distributed as $\mathrm{F}_{\mathrm{k}-1, \mathrm{~N}-\mathrm{r}}$ under $\mathrm{H}_{\mathrm{o}}: \mathrm{Q}^{\prime} \beta=\theta$.

To illustrate, suppose we wish to test the proposition that all the regression weights $\mathrm{w}_{\mathrm{k}}^{*}$ are equal in Formula 3.5. This is equivalent to testing whether the unweighted case of the attraction paradigm is all that is required by the data. For $m$ attitude items, and therefore $m+1$ parameters in $\beta$ altogether, the hypothesis

$$
\mathrm{H}_{\mathrm{o}}: \mathrm{w}_{1}^{*}=\mathrm{w}_{2}^{*}=\ldots=\mathrm{w}_{\mathrm{m}}^{*}
$$

can be written as a "linear hypothesis":

$$
\mathrm{H}_{\mathrm{o}}:\left[\begin{array}{rrrrr}
0 & 1 & -1 & & 0 \\
0 & 0 & 1 & -1 & \\
0 & & & & \cdot \\
0 & & & 1 & -1
\end{array}\right]\left[\begin{array}{l}
\mu \\
\mathrm{w}_{1}^{*} \\
\cdot \\
\mathrm{w}_{\mathrm{m}}^{*}
\end{array}\right]=\left[\begin{array}{l}
0 \\
0 \\
\cdot \\
0
\end{array}\right]
$$

This defines $\mathrm{Q}^{\prime}$, and thus $\mathrm{T}(\gamma)$, for testing $\mathrm{H}_{\mathrm{o}}$. Since the theory of the GLM is well known and available elsewhere (e.g., Searle, 1966, 1971; Seber, 1966), we shall not belabor the details of such tests further. A numerical illustration of such tests is given in (Schönemann et al., 1975).

The availability of such a statistical formulation, in our judgment, greatly enhances the utility of the ByrneRhamey formula as a research tool because it provides a simple and efficient machinery for estimating the weights and replaces inspection and intuition with clear-cut and well-understood statistical tests.

\section{REFERENCES}

Bцохом, B. Individual differences in multidimensional scaling. Research Bulletin 68-45. Princeton, N.J: Educational Testing Service, 1968.

BYRNE, D. The attraction paradigm. New York: Academic Press, 1971.

Byrne, D., London, O., \& Griffitt, W. The effect of topic importance and attitude similarity-dissimilarity on attraction in an intra-stranger design. Psychonomic Science, 1968, 11, 303-304.

Byrne, D., \& Nelson, D. Attraction as a linear function of proportion of positive reinforcements. Journal of Personality and Social Psychology, 1965, 2, 884-889.

Byre, D., \& Rhamey, R. Magnitude of positive and negative reinforcements as a determinant of attraction. Journal of Personality and Social Psychology, 1965, 2, 884-889.

Carroll, J. D., \& Chang, J. J. Analysis of individual differences in multidimensional scaling via an $\mathrm{N}$-way generalization of "Eckart-Young" decomposition. Psychometrika, 1970, 35, 283-320.

Clore, G. L., \& BALDRIDGe, B. Interpersonal attraction: The role of agreement and topic interest. Journal of Personality and Social Psychology, 1968, 9, 340-346.

Horan, C. B. Multidimensional scaling: Combining observations when individuals have different perceptual structures. Psychometrika, 1969, 34, 139-165.

SEARLE, S. R. Matrix algebra for the biological sciences. New York: Wiley, 1966.

SEARLE, S. R. Linear models. New York: Wiley, 1971.

SEBER, G. A. F. The linear hypothesis: A general theory. London: Griffin, 1966.

SChönemanN, P. H. An algebraic solution for a class of subjective metrics models. Psychometrika, 1972, 37, 441-451.

Schönemann, P. H., Byrne, D., \& Bell, P. A. Mathematical aspects for a model for interpersonal attraction. Mimeo, Purdue University, 1975.

Wang, M. M., \& Schönemann, P. H. General linear model and MANOVA. Program Manual G4 LMANOVA, Purdue University, 1970.

(Received for publication November 26, 1976.) 